\title{
Enfoque Polifásico Para La Identificación de Alternaria Spp. Aisladas de Alimentos
}

\author{
Lucía da Cruz Cabral (I), Kristian Fog Nielsen (II), Sebastián \\ Stenglein (III), Virginia Fernández Pinto (I), Andrea Patriarca (I) \\ (I) FCEN UBA - Departamento de Química Orgánica (Ciudad Universitaria, Pabellón II, Buenos \\ Aires, Argentina), (II) DTU - Systems Biology, Technical University of Denmark (Lyngby, \\ Denmark), (III) UNCPBA - Laboratorio de Biología Funcional y Biotecnología (Facultad de \\ Agronomía, Azul, Buenos Aires, Argentina)
}

\section{Resumo}

Alternaria es un contaminante frecuente de cultivos, capaz de producir micotoxinas. Abarca una gran diversidad morfológica y ha habido muchos intentos para organizarlo en subgéneros. La clasificación de Simmons (2007) divide el género en 14 grupos-especie (g-e) según su patrón de esporulación en condiciones estandarizadas. Debido a la plasticidad de estos patrones entre especies, es fundamental realizar un enfoque polifásico para lograr una identificación precisa. Se identificaron 45 cepas de Alternaria aisladas de alimentos (14 de tomate, 14 de pimiento, 15 de trigo y 2 de arándanos) según Simmons (2007). La determinación de metabolitos secundarios se realizó en DRYES (14 días a $25^{\circ} \mathrm{C}$ ), seguido de extracción a micro escala, según Andersen y col. (2009). La detección se realizó en HPLC-DAD acoplado a EM ESI-TOF. Se amplificó una región del gen de endopoligalacturonasa utilizando cebadores específicos (endoPG3 y endoPG2b) y se secuenció el fragmento amplificado. Los aislamientos se identificaron por morfología como pertenecientes al g-e A. tenuissima (29/45) y A. arborescens (11/45). El resto de las cepas presentó características intermedias entre estos grupos y el g-e A. alternata, y se clasificaron como Alternaria spp. Por análisis del perfil de producción de metabolitos secundarios no se encontró ningún compuesto representativo de grupo, presentando todos los aislamientos perfiles superpuestos. El estudio de las secuencias del fragmento de endoPG tampoco mostró

\footnotetext{
Referência:

Lucía da Cruz Cabral, Kristian Fog Nielsen, Sebastián Stenglein, Virginia Fernández Pinto, Andrea Patriarca.Enfoque Polifásico Para La Identificación de Alternaria Spp. Aisladas de Alimentos. In: Anais do 12 Congresso Latinoamericano de Microbiologia e Higiene de Alimentos - MICROAL 2014 [= Blucher Food Science Proceedings, num.1, vol.1]. São Paulo: Editora Blucher, 2014. DOI 10.5151/foodsci-microal-043
} 
características distintivas dentro de cada grupo. La comparación de las secuencias con bases de datos (BLAST-NCBI) mostró superposición y alto porcentaje de similitud (> 90\%) con especies dentro de estos tres g-e. La correcta identificación de hongos a nivel de especie es importante ya que hace posible predecir la producción de metabolitos secundarios y su comportamiento fisiológico. Desde el punto de vista de la seguridad alimentaria, el conocimiento del patógeno permite evaluar el riesgo y desarrollar estrategias de prevención. El enfoque polifásico utilizado es una estrategia abarcativa que incluye diferentes aspectos de la fisiología del hongo en estudio. Las herramientas usadas en este trabajo no fueron suficientes para segregar las diferentes especies de Alternaria de esporas pequeñas actualmente reconocidas (Simmons 2007). La taxonomía de Alternaria se encuentra actualmente en revisión.

Palavras-Chave: Alternaria, Micotoxinas, Enfoque polifásico, Tomate, Trigo

\section{Agência de Fomento:}

